\title{
Neurosurgery - A Medical student's perspective on its emotional toll
}

\author{
Vishnu Vardhan Byroju and Satyajit Patra* \\ Division of Biochemistry and Genetics, American International Medical University, Gros Islet, Saint Lucia, West Indies
}

While I was rotating in the department of neurosurgery of a teaching hospital in India, I've realized surgeons had to work with situations of trying to break bad news to patients and cope up with their own apparent failures. Neurosurgery has been an area where doctors would recommend students to reconsider owing to its very nature of poor surgical outcomes. The emotional pain to explain pitfalls of surgery to a patient and save every functional aspect of their brain is often tough to spectate.

As a case in point, a 37y/o female was brought as a referral from the neurologist because of a suspected tumor lying right in the midline and attached to the dura as seen in the radiographs. The surgery was scheduled and the patient was ready for the stress and surgery, which oftentimes, the patients seem to take as one. The surgeon had to explain the possibility of losing a limb to the patient which, for the medical fraternity, was a rather simple understanding of homunculus and location of tumors and how they affect the individual. But for her and her family, it was not so simple. They didn't seem to understand the reason of her losing a limb's function which was completely fine. The patient needed to know if there was truth in this, or to say, the 'negative predictive value' of surgeons' words. After clarifying to the patient, high risk surgery consent was obtained duly and the patient was prepared for surgery.

It was the day of surgery. The surgeon marked the incision site, final checks with everyone in the OR were made and anesthesiologists started. The patient was intubated and injected with sedatives and amnesia was induced, creating a sense of calm in the OR. The surgeon meticulously incised the skin layer and continued through the fascia till the skull was visible. Bur, which looked similar to a hardware drill tool, was brought in to the sterile field and four entry points were made through the skull. The bone flap was removed and placed in saline suspension. The dura was incised making sure the layer wasn't destroyed soon after which cautery was running through the brain, with caution to be as far as it can from the central sulcus while still making sure that the tumor is reached. The tumor was located after about fifteen minutes and it didn't look any different to me, a $3^{\text {rd }}$ year medical student. It was all the same, brain tissue covered in blood, with instruments around it, variegated colors seen at various sites until the surgeon pointed towards the clear margins. With a tumor holder, biopsy was obtained and sent to the pathology department for a freeze fraction preparation to identify the specimen.

The report reached soon enough, and the moment I saw it, I was trying to remember the tumor's whorled pattern of cells that I learned in my neuroscience classes. The pathology report, confirming it as a meningioma, made me happy which I realized soon enough wasn't the emotion an OR would be welcoming. The surgeon was satisfied that enough of tumor tissue had been removed and any further instrument use might cause damage. After performing necessary checks such as counting gauze pads, a gelatin based hemostatic colloid was placed and dura was sutured, making sure the tension created was suitable. The bone flap was placed over the dura and locked into position with cross sutures. The incision was closed in layers and the residents took over the completion after which the OR staff soon resumed work of cleaning up the mess surgeries often create. Shifted to the hospital bed, the patient was conscious after the necessary post-operative measures were taken and the resident was ready for questions.

I was following the surgeon around and reached the patient's room where he asked to raise her right leg up straight which she was able to perform. Fearing the complications of surgery, he asked her to do the same with her left leg which did not have a reasonable result. I could sense the despair as the surgeon wasn't ready to accept defeat. After completing the sensory examination, he asked her to raise her left leg again which, despite her visible struggle, was stationary. We left the patient's room, informing them that we are going to be back in a short while to reassess.

The surgeon was preoccupied with lots of things now, evident by the context (which was all about meningioma) of our conversation and the despair in his tone. After half an hour in the OR to make sure everything to ensure sterility for the next case was going right, we found ourselves in the same situation again. The surgeon made another attempt at the patient but with nothing to change and he now had the responsibility to let the attendants know that their beloved might have lost function of a limb. The tumor extraction had damaged the tissue or the vasculature around the midline motor cortex

With empathy in his tone, he delivered the news while providing hope that in some cases, the limb gains function in some time, say 6 months, with physiotherapy and the likes. We left the intensive care after the surgeon let the nurses know what's next. As I tried to converse with him about the next step, he was preoccupied with meningioma. That was the only thing he was concerned about. Quarter hour passed while he updated the surgery notes as I was reading it. While I was absorbed into the drawing he was making, he picked up his phone and made a call to the chief informing him that the patient they operated on lost functionality of the left limb and that he would reassess her during

${ }^{\star}$ Correspondence to: American International Medical University, Gros Islet, Saint Lucia, West Indies, E-mail: dr.patra@aimu-edu.us

Received: October 18, 2019; Accepted: November 08, 2019; Published: November 12, 2019 
a follow up. It all seemed to happen so quickly, it convinced me that everything was alright with the surgeon till what he spoke to me about next.

"This is how it usually is. This is what we call a bad day in the field. Not even a double duty would make us feel this way. I shouldn't have told the chief what happened which obviously wouldn't have been the best of decisions on my part. Now, his day is ruined".

What could possibly convince a chief surgeon that a patient outcome which was already expected turned out to be true? What could have helped his mood for the day? I was sure that the chief wasn't the only one in a bad mood now. After a few minutes of silence, the surgeon opens up again.

"This is what I don't like about my field. No matter what we do, sometimes it never works. It's then our responsibility to explain to patients what has gone wrong and why it is that way. It's always a look of surprise they give us when we tell them we can't give them a definite prognosis." The surgeon had something to be unhappy for the rest for the day.

Another 18 y/o male was brought in for a MVA (motor vehicle accident) was operated on for his epidural hematoma. Although he had a history of mild alcoholism, his waking up after the surgery in confusion, extreme agitation, psychosis and paranoia could not be explained by the team. Attempts to restore his cognition in the following days included IV fluids and antipsychotic medications which failed. The patient had to be shifted to the department of psychiatry for further management.

Another case of a $23 y / 0$ female presenting emergently with a case requiring an acute surgical decompression of the spine due to cauda equina syndrome resulting in outcomes that weren't expected was witnessed by the team. Such scenarios often resulted in emotional stress for the surgeons as the failure rate is high.

Neurosurgery has not seen many happy patients in tertiary care. Despite relentless efforts in surgical techniques, knowledge of neuroanatomy and perfecting care over the years, surgeons find themselves in a dilemma when a case has an unexpected outcome. Young neurosurgeons in training are often critical about their patients and intellectualize the cases rather than emotionally indulging in it to avoid its emotional impact. Perhaps surgeons can be benefited by addition of discussions about coping up with the emotional stress they all go through during their training and knowing their colleagues feel the same.

"The operating is the easy part, you know,' he said. 'By my age you realize that the difficulties are all to do with the decision-making." Henry Marsh, Do No Harm: Stories of Life, Death, and Brain Surgery.

Copyright: $\odot 2019$ Byroju VV. This is an open-access article distributed under the terms of the Creative Commons Attribution License, which permits unrestricted use, distribution, and reproduction in any medium, provided the original author and source are credited. 